\title{
Reviewers for Volume 23
}

The following colleagues have generously given of their time and energy to review papers for Law and Human Behavior in the last year. We are grateful for their contribution to this journal and the discipline. Asterisks indicate members of the 1998 editorial board; the list is not exhaustive of the board.

Julie A. Allison

Michael Anch

Paul S. Appelbaum*

Hal Arkes

Donald N. Bersoff*

Eugene Borgida*

Randy Borum*

Brian Bornstein

Bette L. Bottoms*

Jack Brigham*

Elizabeth C. A. Brimacombe

Angela Brown

Maggie Bruck

Kay Bussey

John Carroll

Stephen J. Ceci

Charles Clark

Margaret Coggins

Mark Costanzo*

David N. Cox

Brian Cutler*

John Darley

Shari Seidman Diamond*

Kevin S. Douglas

Joel Dvoskin

John Edens

Donna Eisenstadt

Phoebe Ellsworth*

David L. Faigman*

Normal J. Finkel*

Louise F. Fitzgerald

\author{
Robyn Fivush \\ Diane R. Follingstad* \\ Adelle Forth \\ Patricia A. Frazier \\ Soloman M. Fulero* \\ Jeffrey Gfeller \\ Judith Gibbons \\ James Gillsinan \\ Jonathan M. Golding \\ Gail Goodman* \\ Jane Goodman-Delahunty* \\ Edith Greene* \\ Alexander Greer \\ Thomas Grisso* \\ Barbara Gutek* \\ Craig W. Haney* \\ Valerie P. Hans* \\ Grant Harris \\ Stephen Hart \\ Richard Harvey \\ Reid Hastie \\ Jeffrey J. Haugaard* \\ Kirk Heilbrun* \\ Larry Heuer \\ Steven K. Hoge* \\ Irwin A. Horowitz \\ Harmon Hosh \\ Linda Hurt \\ Saul Kassin* \\ David Kenny \\ Norbert L. Kerr
}




\begin{tabular}{ll} 
Margaret Bull Kovera* & M. D. Robinson \\
Geoffrey Kramer & Ron Roesch \\
Janet Kuebli & Richard Rogers \\
Donna LaVoie & V. Gordon Rose \\
Michael Leippe* & David Ross* \\
Avraham M. Levi & Barry Ruback* \\
Murray Levine & Michael Saks \\
Rod C. L. Lindsay* & David A. Schkade \\
Dan Linz & Regina Schuller* \\
Marsha Liss* & Ralph Serin \\
Elizabeth Loftus* & Christopher Slobogin* \\
James Luginbuhl* & Mark Small \\
Rob MacCoun & Vicki Smith* \\
Roy Malpass & Siegfried L. Sporer \\
Gary B. Melton* & Loretta Stalans* \\
John Monahan* & Henry J. Steadman* \\
Gary Moran & Nancy Steblay \\
Douglas Mossman & Margaret Stockdale \\
Edward P. Mulvey* & Dennis P. Stolle \\
Douglas Narby & Christina Studebaker \\
Michael T. Nietzel* & Janet Swim \\
Maureen O'Connor & William Thompson \\
James Ogloff & Michael Toglia \\
Randy Otto* & Alan Tomkins* \\
Janat Parker & John Turtle \\
Jeffrey Pfeiffer & Tom Tyler* \\
Keri Pickel & Neil Vidmar* \\
Steve Porter & Mellissa Warren \\
Norman Poythress & Gary L. Wells* \\
John B. Pryor & Audrey Wiener \\
Jeffrey J. Rachlinski & Dean Wiley \\
Richard Rakos & Roselle Wissler \\
J. Don Reid* & Stephen Wong \\
N. Dickson Reppucci* & Lawarence Wrightsman \\
Marnie Rice* & Daniel Yarmey \\
Jennifer K. Robbennolt & John C. Yuille \\
& \\
\hline &
\end{tabular}

